\title{
ON INTERACTION CLASSIFICATION ${ }^{1}$
}

\author{
JAMES H. LEIGH AND THOMAS C. KINNEAR \\ The University of Michigan
}

\begin{abstract}
Further classification is made of Lindquist's dichotomy of interaction effects. The extension hopefully reduces errors of interpretation and provides a simple, accurate means of summarizing interactions obtained.
\end{abstract}

A thorough review of the textbooks and reference manuals on experimental design reveals an absence of any discussion of interaction effects which extends beyond the customary treatment of computational requirements, necessary assumptions, and general interpretation. The purpose of this paper is to supplement these design-oriented treatments with the discussion of an analysis-oriented issue: interaction classification.

\section{Theoretical Basis}

Lubin made reference to Lindquist's (1953) distinction between "two important subclasses of significant interaction effects: the 'ordinal' case, where the rank order of the treatments is constant; and the 'disordinal' case, where the rank order of the treatments changes (Lubin 1961, p. 808)." If the cell means relevant to a particular interaction are plotted on a graph with the levels of one factor on the abscissa and the dependent variable on the ordinate axis, disordinal interaction is characterized by crossed lines connecting treatment combinations for each profile displayed (Glass and Stanley 1970, p. 411). The rank order is non-monotonic between two or more adjacent abscissa factor

\footnotetext{
1 The authors appreciate the valuable input from an anonymous referee concerning an earlier draft of this manuscript.

Copyright $\odot 1980$ by Educational and Psychological Measurement
} 
levels. Glass and Stanley specify further that ordinal interaction is apparent in those cases where the lines do not cross. This condition occurs only when the rank order of levels of the factor displayed remains constant over all levels of the abscissa factor.

These authors point out, however, that some interactions may be ordinal with one factor as the abscissa and disordinal with the other. For this reason they caution that the terminology relates solely to properties of graphs. We agree strongly with their premise and suggestion, but we also believe students and casual researchers may continue to identify interactions incorrectly unless further classification of interaction types is made. We offer the following three categories: pure ordinal, hybrid and pure disordinal interactions. An example of each type is displayed in Figure 1.

Pure ordinal interaction characterizes the situation in which, regardless of the factor used as the abscissa, a consistent rank order relationship exists for levels of a factor or factor combination between levels of the abscissa factor. The "slopes" of all lines representing profiles vary in the same direction between adjacent abscissa levels on every possible graph depicting the interaction. This definition does not require that the slopes are always positive or negative across levels of every possible abscissa factor; rather, pure ordinal interaction is a restricted form of ordinal interaction that includes only those cases in which every graph that depicts the interaction is ordinal.

Hybrid interaction differs from pure ordinal interaction in that the
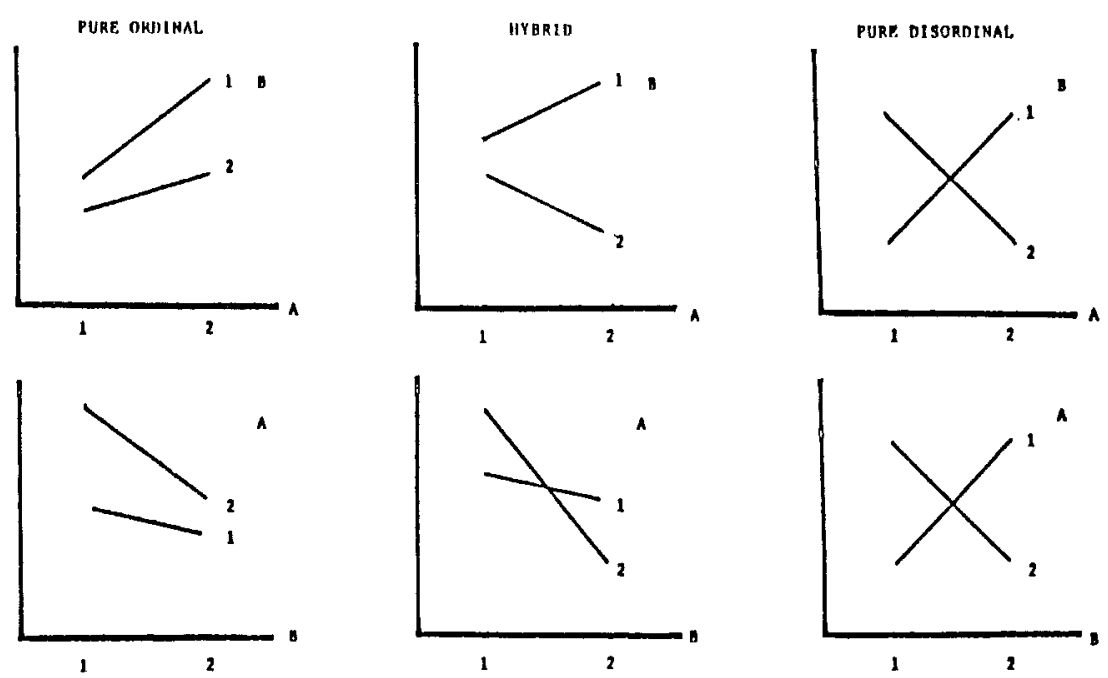

Figure 1. Examples of Three Types of Interaction. 
rank order of treatment profiles is invariant between levels of one or more factors, and it varies between levels of one or more remaining factors. The slopes of two or more lines will vary inversely between two or more levels of any of the possible factors, but they do not necessarily have to cross. Because this inverse relationship implies that in a two-factor interaction contrast the largest and smallest mean values occur at the same abscissa level, the lines must also cross on the graph in which the other factor serves as the abscissa. This relationship will exist for higher-order interactions in which at least one graph will display crossed lines for the interaction contrast and at least one graph will display uncrossed lines; the exact number of each depends on effects present.

Pure disordinal interaction occurs only when a non-monotonic relationship characterizes at least one possible contrast for each factor combination. Regardless of the factor used as the abscissa, lines connecting treatment combinations for two levels of the other component(s) cross at least once. For a particular pure disordinal contrast, the contribution to variation explained made by the interaction parameter is much larger than main effect contributions, and it can be absolute (Lubin 1961, p. 815).

From any of the possible graphs describing a particular situation, it is relatively easy to detect that pure ordinal interaction does or does not characterize the relationship under consideration. However, if the rank order varies between levels of the abscissa factor, the researcher should either construct the remaining possible graphs, investigate the planar relationships in hypergeometric space (for higher order interactions), or compute main effect and interaction contributions to variation explained. In this case, the two-dimensional graph is preferred as an exploratory tool because it is easily constructed and read. It should not, however, serve as a substitute for other means of analyzing relationships.

\section{REFERENCES}

Glass, G. V. and Stanley, J. C. Statistical methods in education and psychology. Englewood Cliffs, NJ: Prentice-Hall, 1970.

Lindquist, E. E. Design and analysis of experiments in psychology and education. New York: Houghton Mifflin, 1953.

Lubin, A. The interpretation of significant interaction. EDUCATIONAL AND PSYCHOLOGICAL MEASUREMENT, 1961, 21, 807-817. 